\title{
Renal Association Clinical Practice Guideline Development Policy Manual
}

\author{
Dr Robert Mactier
}

Consultant Nephrologist and Lead Clinician, Renal Services, NHS Greater Glasgow and Clyde and NHS Forth Valley and Chair of Renal Association Clinical Practice Guidelines Committee

\section{Key Words}

Guideline development manual · systematic review modified GRADE system

\section{Introduction}

\subsection{Clinical Practice Guidelines and the Renal Association}

The Clinical Practice Guidelines Committee was established by the UK Renal Association (RA) to prepare guidelines for the management of patients with renal disease and help identify the data to be collected by the Renal Registry. The Clinical Practice Guidelines Committee is one of three committees reporting to the Clinical Affairs Board of the RA (the others being the Clinical Services Committee and Renal Registry Committee). The Clinical Affairs Board, chaired by the Clinical Vice President, meets three times per annum to overview the activities of its committees and reports to the President and RA Executive (see Figure 1).

\subsection{History of the Renal Association guidelines}

The RA has produced guidance on best practice in the management of patients with kidney disease since 1995 .

1st edition 1995

2nd edition 1997

3rd edition 2002

4th edition 2007-2009

5th edition 2009-2012
The RA guidelines were published as 'clinical standards' for the first three editions (1995-2007) before converting to a standard modular format for the 4th edition in 2007-2009 and the 5th edition which commenced in January 2009. From the 4th edition the Clinical Affairs Board deemed it more appropriate to term the RA guidance 'Clinical Practice Guidelines' rather than 'Clinical Standards'. This change in terminology indicated that the guidance should be used to promote best practice and facilitate clinical judgment without being perceived as prescriptive. For the 4th edition of the RA guidelines the supporting evidence for the clinical practice recommendations was qualified as 'Evidence' when based on randomised controlled trials or systematic reviews or 'Opinion' for all other lower levels of evidence. An archive of these editions is available on the old guidelines page of the website: http://www.renal. org/Clinical/OldGuidelines.aspx

For the 4th and 5th editions the guidance on key subject areas in nephrology, dialysis and transplantation was divided into several sections (modules) rather than produced as a single document. These modules were devised using an evidence-based approach and contained a number of recommendations with audit measures, supporting rationale and references. The 4 th edition consisted of five modules and was completed in 2008. The number of guideline modules was expanded to 14 for the 5th edition: http://www.renal.org/clinical/ GuidelinesSection/Guidelines.aspx

As much of the evidence to support the recommendations in Clinical Practice Guidelines comes from

\section{KARGER \\ Fax +41613061234 E-Mail karger@karger.ch} www.karger.com
Dr Robert Mactier

Email: robert.mactier2@ggc.scot.nhs.uk 


\section{The Renal Association}

\section{TRUSTEES}

\section{EXECUTIVE COMMITTEE}
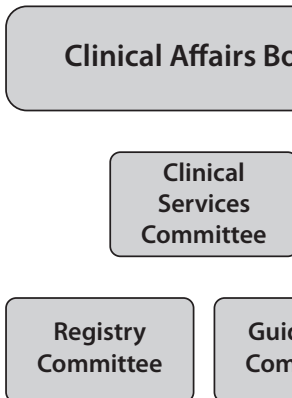

Figure 1

observational clinical studies rather than randomised controlled trials (RCTs) or systematic reviews the modified GRADE (Grades of Recommendation, Assessment, Development and Evaluation) was introduced in the current 5th edition to provide a transparent assessment of both the strength of recommendations and level of evidence. The use of GRADE has been adopted by other national and international guideline development groups in nephrology e.g. Kidney Disease Improving Global Outcomes (KDIGO), European Renal Best Practice (ERBP) and Caring for Australians with Renal Insufficiency (CARI).

The main target audience for the RA guidance is the entire renal community caring for patients with kidney disease within the UK healthcare system. The key professional groups include: renal medical staff (consultants, associate specialists, specialty doctors and specialty trainees), renal nursing staff especially specialist renal nurses working in dialysis, transplantation and patient education, renal technical staff, and all other health professionals caring for patients with renal disease (e.g. renal dieticians, renal pharmacists, social workers).

\subsection{Aims and structure of the guideline development manual}

There have been a number of stepwise developments in producing the RA Clinical Practice Guidelines for the 4th and 5th editions of the guidance and consequently the main aims of this manual are:

- to combine the range of improvements introduced into the guideline development process in recent years into a single document

- to develop a reference tool for current and future co-authors of the guideline modules

- to summarise the guideline process for all users of the guidance but especially for members of the RA, stakeholders, patients and sister agencies.

Based on the Appraisal of Guidelines for Research \& Evaluation (AGREE) Instrument [1] the subsequent sections of this policy document demonstrate that the Clinical Practice Guidelines of the RA are:

- produced to promote good kidney care and reduce the burden of chronic kidney disease and acute kidney injury

- produced by kidney specialists and other healthcare professionals caring for patients with kidney disease for the benefit of peer healthcare professionals, patients and the public

- produced using a transparent, consistent and reliable development process

- designed to provide recommendations based and graded on the best available evidence

- designed to provide recommendations - strong or weak - weighing up the cost, burden and benefits of treatment or intervention

- designed to provide audit measures for the guideline recommendations

- recognised by KDIGO and other international nephrology guideline development groups.

\subsection{Review and update of the guideline development manual}

The guideline development process and publication format have changed for the past two editions of the RA guidance and future modifications and improvements are anticipated. It is planned that this manual will be updated every three years corresponding to the maximum time interval for updates of the guidance.

\section{Selection and planning of guideline modules}

\subsection{Selection criteria for guideline modules}

The Clinical Practice Guideline recommendations within the modules of the 4 th and 5 th editions cover 
the main topic areas and agendas raised by the National Service Framework (NSF) for Renal Services Part 1: Dialysis and Transplantation and Part 2: Chronic Kidney Disease, Acute Renal Failure and End of Life Care in England and National Health Service - Quality Improvement Scotland (NHS QIS) in Scotland [2-4]. Both the NSF for Renal Services and NHS QIS highlight the need to identify markers of good practice to deliver equity of access, patient choice and a high quality of care in nephrology, dialysis and kidney transplantation particularly on treatments, interventions, technologies and resources needed to provide high quality care for acute and chronic kidney disease and optimise patient outcomes on dialysis and kidney transplantation. Consequently the topics for the modules of the 5th edition of guidance were selected to cover all of the main areas of clinical management of patients with renal disease identified by the Department of Health and NHS Scotland as well as the RA. The topics for guidance are submitted to and approved by the Clinical Affairs Board before being placed on the RA website: http:// www.renal.org/Clinical/FutureClinicalPracticeGuidelines. aspx

In addition specialist areas of guidance which will require development in collaboration with other specialist societies undergo approval by the Clinical Affairs Board and then proceed through the same process of guideline development and peer review.

The guidance contained within the full range of guideline modules provides an up-to-date template for the management of renal disease within the UK healthcare system and with the use of audit measures serves to define the dataset collected by the UK Renal Registry as well as for local and regional audit. The close links with the UK Renal Registry promote implementation of the guideline recommendations and the achievement of performance indicators. The main objective of the Clinical Practice Guidelines is to improve clinical practice in renal services nationwide and it should be noted that the Renal Registry has reported progressive improvements in kidney patient outcomes in recent years: www.renal.org/Clinical/RenalRegistry.aspx

\subsection{Timelines for development of guideline modules}

The dates of planned guidelines are published on the Future guidelines page of the RA website.

Dates covered by the initial literature search performed in the preparation of the guidance should be recorded in the introduction section of the modules. Each module requires in excess of four months for completion after the first draft is prepared to allow 1 month for feedback from the first and final drafts, the preparation of the final draft and final version to take account of feedback and endorsement of the final version by the Clinical Affairs Board. If significant new data from high quality studies becomes available during the production of a guideline module on the same subject area the recommendations in this guideline can be adapted for the next version.

For current guidelines the date of completion of the current guideline and the date for updating the guidance are clearly displayed on the RA website main guidelines page: http://www.renal.org/clinical/GuidelinesSection/ Guidelines.aspx

In addition the dates of the first and final drafts are recorded on the website in the archived PDF versions at the foot of the current guideline.

\subsection{Updating of existing guideline modules}

The dates of planned updates of existing guideline modules are published on the main guidelines page of the website with the intention that updates of existing guidelines should be available within 3 years. If important new information from high quality studies becomes available prior to planned updates the electronic website modular format permits recommendations to be changed if this is deemed appropriate and endorsed by the Clinical Affairs Board.

\subsection{Composition and responsibilities of the guideline development group}

The co-authors of each guideline have expertise, often at the national level, in the subspecialty field in which they are invited to produce clinical guidance. The coauthors of each module are identified well in advance of preparation of the guidance and are selected because of their expertise and track record of interest in the subspecialty area and freedom from overt conflicts of interest: http://www.renal.org/Clinical/FutureClinical PracticeGuidelines.aspx

Each guideline module has two lead co-authors who may invite colleagues with specialty knowledge and expertise to help produce the guidance. One of the coauthors is identified as the senior author with responsibility for timely preparation of the guideline: he/she will step down for the next planned update and the second co-author will prepare the next guidance with one or more new co-lead(s). This approach helps to maintain continuity whilst extending expert peer involvement and so assures the quality of future guidance. 
All co-authors of the current guidance or guidance in preparation are members of the Clinical Practice Guidelines Committee. As there are more than 28 career grade nephrologists serving as expert co-leads for the 14 guideline modules of the 5th Edition of the guidance the Clinical Practice Committee represents more than $5 \%$ of the consultant nephrologists within the RA. The involvement of a significant proportion of the UK renal specialists in the production of the guidelines promotes wider acceptance and credibility for the guidance issued to peer professionals. In the preparation and publication of the guidance the Committee is responsible to the Chairman who in turn is responsible to the Clinical Affairs Board and Executive. The Chairman is a member of the Executive of the RA (see Figure 1) and the term of office for the Chairman is 3 years with the option for the Executive to extend the post for 1 year to maintain continuity or complete work in progress.

\subsection{Declaration of conflicts of interest}

All co-authors are required to declare their potential conflicts of interest in the acknowledgements at the end of each module. None of the co-authors have, or will acquire, any financial gain from developing the recommendations in their guideline module.

\subsection{Funding of guideline development}

The RA was founded in 1950, is registered in England and Wales as Company 2229663 and is registered as charity number 800733 .

The RA Clinical Practice Guidelines are not funded by any external organisation, commercial company or charity. The co-authors of each module are selected for their expertise in the subspecialty area and because they are free of overt conflicts of interest with major commercial providers of renal replacement therapy technology, disposables and drugs.

The Clinical Practice Guideline Committee receives no funding apart from minor expenses from the RA Executive to cover the cost of an annual meeting (held during the Annual Meeting of the RA), and the secretarial costs for typesetting the draft and final versions of the guideline modules.

\section{Development process of the guideline modules}

The RA guidelines are developed using an explicit methodology based on five core principles:
- development is carried out by nationally representative experts in the field of guidance who are free of overt conflicts of interest

- the expert group performs a systematic search to identify and critically appraise the evidence

- recommendations using the GRADE system are explicitly linked to the supporting evidence

- recommendations take account of equality issues, financial and resource implications and patient choice and lifestyle

- recommendations are open to two rounds of peer review by the full membership of the RA, stakeholders, patients and interested members of the public before being submitted for approval by the Clinical Affairs Board.

\subsection{Selection criteria of topics within modules}

Each new guideline module is approved by the Clinical Affairs Board prior to beginning the process of producing the guidance. The current guideline modules cover the main areas in nephrology, dialysis and kidney transplantation with the main aims of reducing morbidity and mortality in patients with renal disease and addressing important differences in the utilisation of drug management, interventional procedures, dialysis and kidney transplantation among kidney units.

The selection of key issues for each guideline is based on clinical priorities, the expert co-authors' knowledge of the available literature, the range of treatment and interventions in this field and outcomes which are important to patients. On this basis several criteria are used by the expert co-authors of each guideline to decide which topic areas within each module merit inclusion in the guidance:

- areas of variation in clinical practice

- areas of variation in patient outcomes

- resources to provide high quality patient care

- interventions, procedures and drug management which influence patient morbidity and/or mortality

- patient safety and avoidance of preventable complications.

The definition of the target population and interventions is an essential component in the development of the guideline recommendations in the management of renal disease and in the published data which provide the supporting evidence for the recommendations. Application of these principles is readily achieved using the PICO framework. 
The patients or population of interest are patients with chronic kidney disease (CKD), patients receiving dialysis (haemodialysis or peritoneal dialysis) or a kidney transplant and patients with acute kidney injury (AKI). The presence of these criteria will largely identify patients for inclusion in literature reviews to generate patient subgroup specific recommendations in the range of RA guideline modules. The RA guidance applies to adolescents and adults with renal disease: separate guidance for children with renal disease is prepared by the British Association of Paediatric Nephrology (BAPN). A link with the guidance on dialysis in children prepared by the BAPN, which was extrapolated from previous RA guidance on haemodialysis in adults, is available in the other guidelines page on the RA website: http://www.renal.org/Clinical/OtherGuidelines.aspx

The guidance is careful not to make recommendations which may prejudice clinical care based on gender, age, ethnicity or socio-economic status. No adult patients with renal disease are excluded from the guidance.

The interventions in the guidance on management of patients with renal disease are readily identified in the literature to generate intervention specific recommendations: different drug treatments for clinical conditions or complications secondary to renal disease (e.g. anaemia, hypertension and cardiovascular disease, mineral and bone disorders, proteinuria in CKD patients) and different forms of renal replacement therapy (e.g. haemodialysis, peritoneal dialysis and kidney transplantation).

The comparisons in the RA guidance are mainly with placebo/no treatment or comparison between different treatment options e.g. drug treatments or dialysis modalities.

Hard outcomes such as patient mortality, morbidity, hospitalisation and complication rates are preferred in developing recommendations within the RA guidance but many studies in nephrology only report surrogate outcomes.

Therefore the co-authors for each guideline are able to identify several subject areas within each guideline (formatted numerically as main headings in the guidance) within which a number of review questions are addressed to allow evidence based recommendations to be formulated (formatted numerically within each main heading in the guidance).

\subsection{Systematic literature review}

The co-authors of each module are identified in advance of the guidance and are selected because of their expertise and track record of interest in the subspecialty area and freedom from overt conflicts of interest: www.renal.org/Clinical/FutureClinicalPractice Guidelines.aspx

The co-authors will have followed the literature in their field for many years prior to undertaking the search for evidence to prepare their guideline module. The RA guidelines do not have the resources to commission and conduct a formal evidence review but the co-authors of each module conduct their own systematic search of the literature published in English just prior to preparing the guideline. The dates covered by the systematic literature search should be stated clearly in the introduction of each module along with specific details of the search strategy and search terms used. This will involve, as a minimum, a search on Pubmed and/or Medline using key search terms documenting the relevant literature for the search terms within the guideline module agreed by the guideline development group as well as a review of the Cochrane Library Database and Clinical Trials database. Articles not available in English or only available in abstract forms, letters, case reports, editorials or review articles are excluded.

The co-authors also review other nephrology guidelines - such as clinical practice guidelines issued by other national and international societies such as Kidney Disease Improving Global Outcomes (KDIGO), European Renal Best Practice on behalf of the European Renal Association (ERBP), Kidney Disease Outcomes Quality Initiative (KDOQI) on behalf of the National Kidney Foundation in the USA or guidelines relevant to the topic such as the anaemia and CKD guidelines published by NICE.

\subsection{Selection and evaluation of the evidence}

The expert co-authors assess articles for relevance to the guideline topic, eligibility for inclusion in the evidence base for that guideline and methodological quality. Articles are considered of particular relevance if they are describing:

- prospective randomised or quasi-randomised trials

- controlled trials

- meta-analyses of several trials

- Cochrane systematic reviews

- systematic reviews.

The guidance producers include all relevant RCTs, systematic reviews and meta-analyses in preparing recommendations and the supporting evidence for the 
rationale of the recommendations. In many areas in nephrology the number of such high quality publications is however relatively low compared with other specialties and much of the supporting evidence in nephrology, dialysis and transplantation is based on observational studies. In general the co-authors do not exclude this evidence from the literature given that the GRADE system provides an informative and transparent means of providing strong or weak recommendations for best practice even if the available supporting evidence is limited to low level evidence such as observational and case-control studies or case reports. In such circumstances the recommendations are qualified explicitly by an appropriate low grading of the level of evidence (grade C or D).

\subsection{Grading the guideline recommendations}

In recent years, the Grading of Recommendations Assessment, Development and Evaluation (GRADE) Working Group has developed a new approach to grading evidence that moves away from initial reliance on study design to consider the overall quality of evidence across outcomes. The GRADE system was developed by an international group of guideline developers and methodologists to maximise the usefulness of clinical practice guidelines in the management of typical patients [5-11].

The advantages of the modified GRADE system are:

1. The grading system provides an informative, transparent summary for clinicians, patients and policy makers by combining an explicit evaluation of the strength of the recommendation with a judgment of the quality of the evidence for each recommendation.

2. The two-level grading system of recommendations has the merit of simplicity.

A Grade 1 recommendation is a strong recommendation to do (or not do) something, where the benefits clearly outweigh the risks (or vice versa) for most, if not all patients. A Grade 2 recommendation is a weaker recommendation, where the risks and benefits are more closely balanced or are more uncertain. Two levels facilitate a clear interpretation of the implications of strong and weak recommendations by clinicians. Explicit recommendations are made on the basis of the tradeoffs between the benefits on the one hand, and risks, burden, and costs on the other.

3. Standard wording is used to indicate the strength of each recommendation.
It is desirable to provide clinicians with a standard terminology to aid interpreting the strength of recommendations.

When making a strong recommendation guideline authors are encouraged to use 'We recommend ...' and when making a weak recommendation authors should use 'We suggest ...'. The use of the active voice attributes responsibility for the recommendations to the guideline authors and their supporting organisation.

For example:

'We recommend that specialist trainees in renal medicine should sit the Knowledge Based Assessment examination before the last two years of training. (1D)'

'We suggest that specialist trainees in renal medicine should become members of the RA to enhance their continuing medical education and support their professional development. (2C)'

4. Explicit methodology is used to describe the quality of evidence.

Grade A evidence means high-quality evidence that comes from consistent results from well-performed randomised controlled trials, or overwhelming evidence of some other sort (such as well-executed observational studies with very strong effects).

Grade B evidence means moderate-quality evidence from randomised trials that suffer from serious flaws in conduct, inconsistency, indirectness, imprecise estimates, reporting bias, or some combination of these limitations, or from other study designs with special strength.

Grade C evidence means low-quality evidence from observational studies, or from controlled trials with several very serious limitations.

Grade D evidence is based only on case studies or expert opinion.

5. Ability to upgrade and downgrade the quality of evidence.

GRADE can appraise all relevant study data to upgrade or downgrade the overall quality of evidence

RCTs $=$ high initial grade

Observational studies $=$ low initial grade

Other evidence $=$ very low initial grade

Reduce grade if study limitations, inconsistency between studies, surrogate outcome but no direct patient outcomes, bias

Raise grade if confounders would have reduced the observed effect, strong association without plausible confounders or large dose-response effect 
The review of critical appraisal tools for use by specialist medical societies conducted by NHS Plus and the Clinical Effectiveness Forum of Royal College of Physicians of London gives support to the use of GRADE in producing guidance in nephrology [12]. An extract from its Executive summary states:

'SIGN, GRADE, GATE and NSF use different methods for grading evidence and recommendations. SIGN uses a '++', '+', '-' system to grade evidence; GRADE uses a 'high', 'moderate', 'low' or 'very low' system; GATE has no pre-defined grading levels; and NSF has a mixture of letters, numbers and words to grade evidence. To grade recommendations, SIGN uses an 'A', 'B', 'C', 'D' system, GRADE uses 'strong' and 'weak' levels; GATE is used to assist the development of recommendations rather than being a prescribed system and so has no levels of grading; and NSF uses an 'A', 'B', 'C' system to grade recommendations.

Each system has strengths and weaknesses depending on the field of research and study design for which it is used. A matrix has been developed to show these strengths and weaknesses and to suggest the most appropriate system to use for the type of study being assessed. Based on the research conducted for this report, SIGN or GRADE have been deemed the more appropriate systems for assessing studies in therapy research; GRADE or NSF have been deemed the more appropriate systems for assessing diagnostic or screening studies; GRADE has been deemed the more appropriate system for causation studies; and NSF has been deemed the more appropriate system for prognosis, psychometric, qualitative and behavioural studies.'

For the above reasons the GRADE approach has gained wide acceptance by guideline developers across the world as a standardised critical appraisal and recommendation system. The modified GRADE system offers the dual benefit of defining the strength of the recommendations of the guideline authors and the level of evidence upon which each of the recommendations is based. This grading system classifies expert recommendations as 'strong' (Grade 1) or 'weak' (Grade 2) based upon the balance between the benefits and risks, burden and cost-effectiveness. The quality or level of evidence is designated as high (Grade A), moderate (Grade B), low (Grade C) or very low (D) depending on factors such as study design, directness of evidence and consistency of results (Table 1).

Most guideline organisations have recognised the need for a standard grading scheme and the GRADE system has been adopted by many leading organisations including NICE, SIGN, BMJ and WHO. It was agreed at the KDIGO meeting on 6th December 2008 to use the modified GRADE system in future KDIGO guidelines and discontinue the use of moderate strength recommendations. KDOQI and European Renal Best Practice have indicated that they will also adopt this system and UpToDate started using this system successfully in 2006.

The meeting of the RA Executive in January 2009 and the Clinical Practice Guidelines Committee meeting on 21st April 2009 ratified the proposal that the modified GRADE system (Grades of Recommendation, Assessment, Development and Evaluation) is used in all updates and new guideline modules from the beginning of 2009 on the basis that this grading system [13].

- provides an estimate of both the quality of evidence and strength of recommendation

- permits the development of guidelines in the absence of randomised clinical trials (RCTs)

- promotes harmonisation of national and international guidelines (e.g. with NICE, KDIGO).

\subsection{Forming the guideline recommendations and audit measures}

The guidance in each module has been designed to:

- provide clear advice and guidance on effective clinical practice

- identify audit measures for review and monitoring of the performance of renal services

- support staff in improving renal services

- promote patient safety and implementation of clinical governance.

The expert co-authors review all of the available evidence in a topic area to make draft recommendations with a supporting rationale. Explicit recommendations are made on the basis of GRADE by assessing the tradeoffs between the health/patient benefit on the one hand, and risks, burden, and costs on the other. The recommendations within the guidance are written with an emphasis on using standard, consistent and up-to-date terminology to avoid ambiguity. This terminology is well established and familiar to the main target audience of the guidance. The recommendations are formulated to be clear, concise and able to be interpreted separately from their supporting rationale. This is reinforced by the publication of a separate summary of the recommendations after the introduction to each guideline to 
Table 1. Summary of the modified GRADE system (Grades 1A-2D)

\begin{tabular}{|c|c|c|c|}
\hline $\begin{array}{l}\text { Grade of } \\
\text { Recommendation }\end{array}$ & Clarity of risk/benefit & Quality of supporting evidence & Implications for clinical practice \\
\hline $\begin{array}{l}\text { 1A } \\
\text { Strong } \\
\text { recommendation. High } \\
\text { quality evidence }\end{array}$ & $\begin{array}{l}\text { Benefits clearly } \\
\text { outweigh risk and } \\
\text { burdens, or vice versa }\end{array}$ & $\begin{array}{l}\text { Consistent evidence from well } \\
\text { performed randomised, controlled } \\
\text { trials or overwhelming evidence of } \\
\text { some other form. Further research is } \\
\text { unlikely to change our confidence in } \\
\text { the estimate of benefit and risk }\end{array}$ & $\begin{array}{l}\text { Strong recommendations, can apply } \\
\text { to most patients in most } \\
\text { circumstances without reservation. } \\
\text { Clinicians should follow a strong } \\
\text { recommendation unless there is a } \\
\text { clear rationale for an alternative } \\
\text { approach }\end{array}$ \\
\hline
\end{tabular}

$1 \mathrm{~B}$

Strong

recommendation.

Moderate quality

evidence
Benefits clearly outweigh risk and burdens, or vice versa

Benefits appear to outweigh risk and

Strong

recommendation. Low quality evidence

$1 \mathrm{D}$

Strong recommendation Very low quality

evidence

\section{A}

Weak recommendation. with risks and burdens

High quality evidence

Benefits appear to

outweigh risk and

burdens, or vice versa
Benefits closely balanced Consistent evidence from well performed randomised, controlled trials or overwhelming evidence of some other form. Further research is unlikely to change our confidence in the estimate of benefit and risk

2B

Weak recommendation. Moderate quality evidence

2C

Weak recommendation. Low quality evidence

2D

Weak recommendation Very low quality evidence

Evidence limited to case studies

\section{Evidence from randomised,} controlled trials with important limitations (inconsistent results, Further research may change the estimate of benefit and risk

Uncertainty in the estimates of benefits, risks, and burdens; benefits may be closely balanced with risks and burdens

Uncertainty in the estimates of benefits, risks, and burdens; benefits may be closely

Evidence from observational studies, unsystematic clinical experience, or from randomised, controlled trials with serious flaws. Any estimate of effect is uncertain

Evidence limited to case studies and expert judgment
Evidence from randomised, controlled trials with important limitations (inconsistent results, methods flaws, indirect or imprecise), or very strong evidence of some other research design. Further research may impact on our confidence in the estimate of benefit and risk

Evidence from observational studies, unsystematic clinical experience, or from randomised, controlled trials with serious flaws. Any estimate of effect is uncertain methods flaws, indirect or imprecise). balanced with risks and burdens
Strong recommendation and applies to most patients. Clinicians should follow a strong recommendation unless a clear and compelling rationale for an alternative approach is present

Strong recommendation, and applies to most patients. Some of the evidence base supporting the recommendation is, however, of low quality

Strong recommendation based mainly on case studies and expert judgment

Weak recommendation, best action may differ depending on circumstances or patients' or societal values

Weak recommendation, alternative approaches likely to be better for some patients under some circumstances

Very weak recommendation; other alternatives may be equally reasonable
Weak recommendation; other alternatives may be reasonable 
serve as a quick reference guide before the full text of the guidance.

The guidance co-authors should ensure that the balance between health benefits and risks/harm is in favour of the former before producing recommendations to follow a specified management. Recommendations not to follow a specified management or treatment should be made if the risks/harms exceed the assessed health gain e.g. the recommendation not to measure serum erythropoietin levels in Guideline 1.4 of assessment of renal anaemia in the anaemia in CKD module.

The modular format with numbering of the subject headings and guideline recommendations within each module has been designed to make the guidance user friendly. The recommendations are collected under headings which define the patient group, treatment or stage of disease to which the recommendations apply. Hence the guidance within different modules or different sections of a module will apply to patients with different categories of renal disease, specific complications of $\mathrm{CKD}$, different modes of renal replacement therapy or acute kidney injury. The 14 modules cover the main subject areas in the management of patients with kidney disease e.g. peritoneal dialysis, haemodialysis, anaemia of CKD, nutrition in CKD.

The prevalence of CKD and the need for renal replacement therapy is higher in ethnic subgroups of the population e.g. Asian, Afro-Caribbean. However the guidance applies equally to all patient groups regardless of race, disability, gender or age.

The RA guidance attempts to harmonise its guidance with other international nephrology guideline development groups whenever appropriate to the UK healthcare system. The international harmonisation of nephrology guidelines is regarded as desirable to improve kidney care worldwide and a comparison of guideline recommendations and audit measures of the KDIGO, RA, CARI, ERBP, CSN and KDOQI is available on the KDIGO website at www.kdigo.com. This website provides a comparison of key areas of the RA guidance with the recommendations and performance measures which have been developed by the guidance producers of the other national and international nephrology guideline groups cited above.

\subsection{Resource implications of the guideline recommendations}

Management of renal disease demands a relatively high level of healthcare resource and finance. The provision of renal services has been limited by resource allocation in the past and resources are finite. The coauthors of each module should draft and agree the recommendations within each module based primarily on clinical effectiveness but the use of resources and cost effectiveness should also be taken into account. The co-authors should produce recommendations to follow any specified management which on balance favours health gain/patient benefit over risk/harm where there is evidence of clinical effectiveness. At the same time the co-authors may produce no recommendations, or recommendations not to follow a specified management if clinical and cost effectiveness is in doubt. The guidance will always make recommendations not to follow a specified form of treatment when the risks/harms exceed the assessed health gain.

\section{Standard format of guideline modules}

\subsection{Layout of the guideline modules}

There is a standard format for all modules of the 5th edition of the RA guidelines as follows:

Title page

Contents page

Introduction (including search methods)

Summary of all clinical practice recommendations

Summary of all audit measures

Rationale of each recommendation or group of recommendations followed by all of the references cited in the rationale

Acknowledgements (including co-authors' conflicts of interest)

This format was discussed and agreed at the annual meeting of the Clinical Practice Guidelines Committee in April 2009. The minutes of this meeting are available at the foot of the main guidelines page on the RA website.

\subsection{Introduction}

In the introduction the guideline authors should indicate the background and rationale for the development of the guideline. Links to prior versions of the guideline and links with the guidelines of other international and national guideline development groups should be described when appropriate.

The search strategy with dates of search, search terminology and methods should be described in the introduction. Harmonisation with the recommendations from other international nephrology guidelines should 
be acknowledged to provide clarity to the guidance user. The method of grading the strength of recommendations and level of supporting evidence should be described. The GRADE system for grading the recommendations was adopted for the 5th edition after discussion and approval at the RA Executive meeting in January 2009 and Clinical Practice Guidelines Committee meeting on 21st April 2009 [13].

\subsection{Summary of recommendations}

A summary of the guideline recommendations is collated to provide a list of all recommendations for ease of review by the user. This section is readily available for printing separately from the full guideline and serves as a quick reference guide.

The guidance is divided into subsection headings and the headings are in bold font to identify clearly the specific clinical or healthcare circumstances that apply to the recommendations. The headings and subheadings are tabulated numerically for ease of reference and clarity of presentation.

\subsection{Summary of audit measures}

Each guideline contains a number of audit measures to assist with implementation of the guidance, promote an improvement in the quality of care and allow comparative audit. The audit measures should be measurable, achievable and serve as evidence based criteria for continuing quality improvement. A summary of all of the audit measures in each module is included before the rationale section of all of the recommendations.

\subsection{Supporting rationale and references for recommendations}

This section provides the rationale and chain of logic for the guidance recommendations. The rationale and references are described separately after each recommendation or subgroup of recommendations to allow for ease of updating and editing. The rationale should provide support for the grading of the recommendations.

\subsection{Acknowledgements and Declarations of Interest}

Significant contributions to the guidance from nephrologists, clinical scientists, patients and other stakeholders should be acknowledged. All co-authors should provide declarations of interest in this section.

\subsection{Electronic publication on the RA website}

The 4th and 5th editions of the guidance are published electronically rather than print. This approach enables more rapid publication, promotes dissemination of the guidance and in the current green era cuts down on paper use.

The guidelines pages on the RA website are paginated to be user friendly:

- current and under review guidelines are published on the main guidelines page

- planned guidelines are published on the future guidelines page

- guidelines produced in collaboration with other societies and the NICE and SIGN renal guidance are published in the other guidelines page

- historical RA guidelines are archived in the old guidelines page

It is planned to publish the 5th edition electronically in Nephron Clinical Practice on completion of all of its 14 guideline modules in late 2010. This publication will be in a chapter format similar to the Annual Report of the Renal Registry, which is already published electronically each year in the same Journal. There will be a separate chapter with a summary of the recommendations from all of the modules which may be downloaded or printed as a easy reference guide to users at no cost.

\section{Consultation and peer review of the guideline modules}

The consultation and peer review process during the preparation of the clinical practice guidelines was agreed at the Clinical Practice Guidelines Committee meeting in 15th May 2008 and the process is documented in detail in the minutes of this meeting published on the main guidelines page on the RA website [14].

External peer review, validation and pre-testing of the recommendations within the guidance of each module is achieved by inviting feedback from the membership of the RA on both first and final drafts of the recommendations.

\subsection{Consensus process for grading of the recommendations}

Based upon the GRADE instrument the co-authors of each module aim to reach a consensus on the strength of recommendation ( 1 or 2 ) and level of supporting evidence $(\mathrm{A}-\mathrm{D})$ as described in detail in the 'Grading the guideline recommendations' section and Table 1 . 
The recommendations for the first draft result from a collective decision reached by informal discussion by the expert co-authors and whenever necessary with input from the Chair of the Clinical Practice Guidelines Committee. The number of expert co-authors of each guideline is too small to support formal consensus methods such as the Delphi technique or nominalgroup technique but a wider consensus is achieved subsequently as a result of peer review of both the first and final drafts of the guidance by fellow professionals, stakeholders and patients. Changes to the grading of the recommendations may be considered after feedback from the first and final drafts of the guidance.

\subsection{Peer review of the first draft of the guideline}

The first draft of the guidance is subject to peer review by all of the membership of the RA and invited stakeholders. The membership of the RA exceeds 1000 people and includes almost all of the renal consultants in the UK. The main steps in peer review of the first draft are:

1. The first draft of new or updated guideline modules is put on the guidelines page of the RA website with a request for comments to be sent by email to the lead authors within a one month timeline.

2. At the same time all RA members (as well as committee members) are informed about this by email by the RA Secretary via a notice within the monthly RA newsletter (eNews) or by separate email.

3. Lead authors will also ask other key stakeholders, including patient representatives with kidney disease, to comment on the first draft within 1 month.

4. Lead authors will collate the comments from RA members and other stakeholders and provide a brief summary of the amendments to be included in the final draft.

5. The lead authors should send the final draft and the summary of comments received and key changes to the Chairman within 1 month of the deadline for receipt of comments on the first draft.

\subsection{Peer review of the final draft of the guideline}

The Chairman of the Clinical Practice Committee arranges for the final draft of the guidance to undergo peer review by all of the membership of the RA and invited stakeholders. The key steps in peer review of the final draft are:
1. The final draft of new or updated guideline modules is put on the guidelines page of the RA website with a request for comments to be sent by email to the lead authors within a 1 month timeline.

2. The final draft and summary of comments/key changes to the first draft is circulated to all members of the committee by the Chairman. However to reduce the workload of the committee 2 members of committee will be asked by the Chairman to review the final draft in detail and give feedback to the lead authors, Chairman and other members of the committee within 1 month.

3. The co-authors of each guideline module invite stakeholders and patients with renal disease to provide feedback on the drafts of guideline modules. If patient feedback influences the final guidance this should be acknowledged, for instance by putting patient acknowledgements at the end of the guideline.

4. After incorporating feedback and comments on the final draft the co-authors of the guideline should submit the final version to the Chairman of the Clinical Practice Committee for final editing within 1 month of the deadline for receipt of feedback from the final draft.

\subsection{Approval of the final version by the Clinical Affairs Board}

The Chairman puts forward the final version for review and endorsement by the Clinical Affairs Board at one of the 3 meetings of the Clinical Affairs Board each year or by email correspondence. Once approved the final version is published and locked in the main guidelines page of the website with a notice of the dates of epublication and planned review within 3 years. The draft versions (and final version of the 4th edition when this is available) are recorded as PDF files at the foot of the final version of the guideline. The timeline to complete the two stage peer review process is at least 4 months for each guideline.

All of the current guidelines have the date of completion of the current guideline and the date for updating the guidance clearly displayed on the RA website main guidelines page. If important new information from high quality studies becomes available (for instance a RCT is published in between planned updates) the electronic website modular format permits recommendations to be changed, when appropriate and endorsed by the Clinical Affairs Board. 


\subsection{Summary of the timeline for the RA Clinical Practice Guideline Development Process}

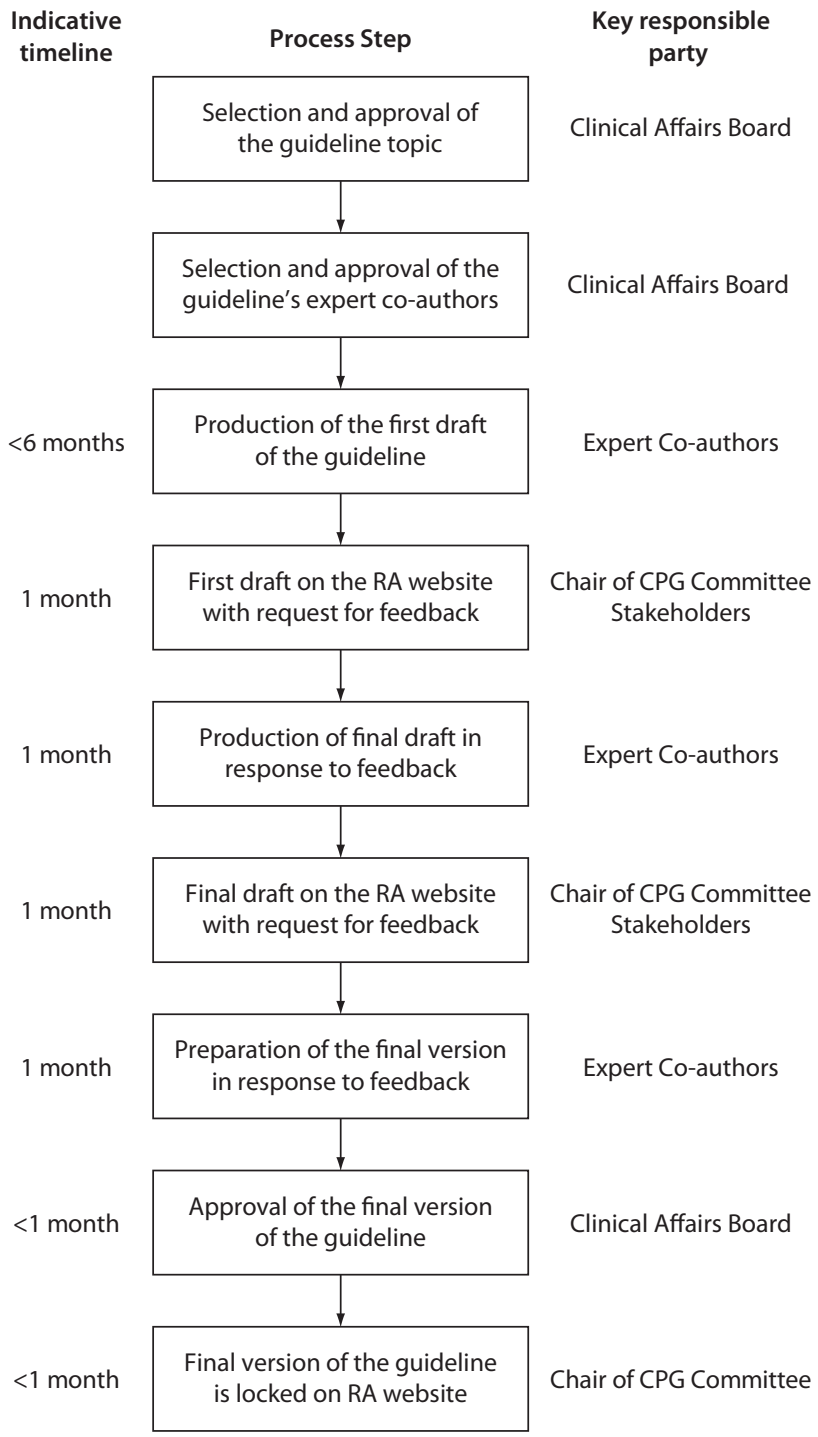

\section{Dissemination and implementation of the guideline modules}

6.1 Notification of epublication of the final version The membership of the RA is notified in the monthly eNews when a final version of a clinical practice guideline is posted on the main guidelines page on the website: http://www.renal.org/clinical/Guidelines Section/Guidelines.aspx

\subsection{Listing of the guidance by NHS Evidence and KDIGO}

After it has been published and locked on the RA website the RA guidance is listed by NHS Evidence in its database of guidance.

A summary of UK RA guideline recommendations and audit measures is available on the KDIGO website at www.kdigo.org. This website also provides a comparison of the recommendations and performance measures of the other international nephrology guidance producers: the European Renal Association (European Renal Best Practice, ERBP), National Kidney Federation in the USA (Kidney Disease Outcomes Quality Initiative, KDOQI), Caring for Australasians with Renal Impairment (CARI) and Canadian Society of Nephrology (CSN). Better links and increased collaboration with KDIGO have led to better dissemination of the guidance worldwide.

The CARI guidelines also include a section describing how their guidance compares with the RA guideline and other nephrology guidelines on the same topic [15].

\subsection{Use of audit measures for national audit by the Renal Registry}

Implementation of the RA guidelines is promoted by the performance of audit on performance measures related to key recommendations within the guidance. The co-authors of each guideline module should identify several audit measures to serve as evidence based useful criteria for continuing quality improvement. A summary of all of the audit measures in each module is included before the rationale section of all of the recommendations.

The audit measures may be used for local and regional audit by individual renal units and all of the renal units within a region. Some of the audit measures are used as performance indicators in national audit performed by the UK Renal Registry. This approach helps ensure that implementation of all of the recommendations covered by national audit is high. Some of the established audit measures have been used as performance indicators by the UK Renal Registry for many years and are utilised to compare the performance of the renal units across the UK in the Annual Reports of the Renal Registry. The latter is published online by Nephron Clinical Practice and on www.renalreg.org

\subsection{Dissemination and implementation initiatives}

Several strategies and initiatives have been introduced to improve dissemination and implementation of the RA guidance:

1. Each guideline module has a summary of recommendations after the contents and introduction. 
This section of the module can be readily downloaded from the website as a concise summary of the recommendations without needing to read, download or print the entire guideline document.

2. A print copy of the Annual UK Renal Registry Report is sent to all members of the RA, to the Clinical Directors of each renal unit and to Chief Executives of each Trust. The Annual UK Renal Registry Report is published on-line in Nephron Clinical Practice and is freely available to all users on www.renalreg.org. For example, the 12th Annual UK Renal Registry Report 2009 was published on-line in Nephron Clinical Practice 2010 and covers the audit and clinical activity until the end of 2008 [16].

3. All of the RA Clinical Practice Guideline modules have been formatted as PDF files at the foot of each module on the main guidelines page of the RA website providing printable copies of each module ready for download from the website at no cost to all users: http://www.renal.org/clinical/ GuidelinesSection/Guidelines.aspx

4. Presentations on the guidance at Continuing Medical Education meetings have been promoted e.g. the draft of the vascular access guidelines and implementation of best practice in vascular access were presented for discussion at one of the CME sessions at the RA annual meeting in May 2010.

5. Epublication is planned in Nephron Clinical Practice on completion of the 14 guidelines of the 5th edition at the beginning of 2011 with a separate chapter providing a summary of all of the recommendations from all of the guideline modules. With this format of publication all of the guideline modules and the chapter providing a summary of the recommendations may be downloaded and printed by any user at no cost. The epublications in Nephron Clinical Practice will be cited by Pubmed and Medline which should promote dissemination of the guidance.

\section{References}

1 The AGREE Collaboration. Appraisal of Guidelines for Research \& Evaluation (AGREE) Instrument. www.agreecollaboration.org

2 The National Service Framework for Renal Services Part One: Dialysis and Transplantation. Department of Health, January 2004

3 The National Service Framework for Renal Services Part Two: Chronic kidney disease, acute renal failure and end of life care. Department of Health, February 2005

4 Clinical Standards, Adult Renal Services. NHS Scotland, February 2002 http://www.nhshealthquality.org/nhsqis/files/Renal_AdultRenalServices _04.pdf

-5 Atkins D, Best D, Briss PA et al; GRADE Working Group. Grading quality of evidence and strength of recommendations. BMJ 2004; 328:1490

6 Guyatt G, Gutterman D, Baumann MH et al. Grading strength of recommendations and quality of evidence in clinical guidelines: report from an American college of chest physicians task force. Chest 2006;129:174-181

7 Guyatt GH, Oxman AD, Vist GE et al. GRADE: an emerging consensus on rating quality of evidence and strength of recommendations. BMJ 2008;336:924-926

8 Guyatt GH, Oxman AD, Kunz R et al. GRADE: Incorporating considerations of resources use into grading recommendations. BMJ 2008;336:1170-1173
9 Guyatt GH, Oxman AD, Kunz R et al. GRADE: going from evidence to recommendations. BMJ 2008;336: 1049-1051

10 Jaeschke R, Guyatt GH, Dellinger P et al. Use of GRADE grid to reach decisions on clinical practice guidelines when consensus is elusive. BMJ 2008;337:327-330

11 Uhlig K, MacLeod A, Craig J et al. Grading evidence and recommendations for clinical practice guidelines in nephrology. A position statement from Kidney Disease: Improving Global Outcomes (KDIGO). Kidney Int 2006;70:2058-2065

12 Baker A, Madan I, Young K, Potter J. A review of critical appraisal tools for use by specialist medical societies. NHS Plus and the Clinical Effectiveness Forum of Royal College of Physicians of London 2008

13 Minutes of the Clinical Practice Guidelines Committee Meeting 21st April 2009. http://www.renal.org/Clinical/GuidelinesSection/Guidelines. aspx

14 Minutes of the Clinical Practice Guidelines Committee Meeting 15th May 2008. http://www.renal.org/Clinical/GuidelinesSection/Guidelines. aspx

15 Acceptance onto Dialysis; Transplantation Nutrition; Living Kidney Donor; Type 2 Diabetes: Kidney Disease; and Renovascular Disease. The CARI guidelines, Nephrology 2010;15(Suppl 1):1-246

16 UK Renal Registry 2009 12th Annual Report. Nephron Clinical Practice 2010;115(Suppl 1):1-370 